\title{
New "Irreducible Wedge" for Scattering Rate Calculations in Full-Zone Monte Carlo Simulations
}

\author{
JOHN STANLEY* and NEIL GOLDSMAN \\ Department of Electrical Engineering, University of Maryland, College Park, MD, 20742
}

\begin{abstract}
To facilitate the implementation of accurate Brillouin zone integration codes for diamond-type semiconductors such as $\mathrm{Si}$ we have constructed a new "irreducible wedge" to be used in conjunction with tetrahedral $k$-space interpolations. This new wedge, defined by the vertices $(000),(100),(110)$ and $\left(\frac{1}{2} \frac{1}{2} \frac{1}{2}\right)$ (normalized to $|X|=1$ ), spans portions of the first three Brillouin zones and contains the equivalent of $1 / 48$ of a Brillouin zone volume. Simple symmetry arguments may be used to establish the equivalency of this new wedge with the standard irreducible wedge.
\end{abstract}

Keywords: Monte Carlo, Brillouin zone integration, irreducible wedge

\section{INTRODUCTION}

Carrier transport investigations based on full-zone band structure require accurate and efficient methods of computing singular integrals over the Brillouin zone. For example, in Monte Carlo simulations, net (integrated) scattering rates and scattering rate densities (in $k$-space) are needed to determine scattering event occurrences and postscattering states for carriers [1]. Such integrals arise from Fermi's Golden rule and are of the general form

$g\left(\varepsilon\left(\boldsymbol{k}^{\prime}\right)\right)=\int d^{3} k M\left(\boldsymbol{k}, \boldsymbol{k}^{\prime}\right) \delta\left\{\varepsilon(\boldsymbol{k})-\left[\varepsilon\left(\boldsymbol{k}^{\prime}\right)+\Delta \varepsilon\left(\boldsymbol{k}, \boldsymbol{k}^{\prime}\right)\right]\right\}$.

${ }^{*}$ Corresponding author
In particular, if $\Delta \varepsilon\left(\boldsymbol{k}, \boldsymbol{k}^{\prime}\right)=0, M\left(\boldsymbol{k}, \boldsymbol{k}^{\prime}\right)=1 / 4 \pi^{3}$ and $\varepsilon^{\prime} \equiv \varepsilon\left(\boldsymbol{k}^{\prime}\right), g\left(\varepsilon^{\prime}\right)$ gives density of states [2]. Integrals of this form usually can only be evaluated numerically, particularly if one is using a full-zone band structure computation for the dispersion relation $\varepsilon(\boldsymbol{k})$. In such cases one usually replaces $\varepsilon(\boldsymbol{k}), M\left(\boldsymbol{k}, \boldsymbol{k}^{\prime}\right)$, and $\Delta \varepsilon\left(\boldsymbol{k}, \boldsymbol{k}^{\prime}\right)$ with appropriate piecewise linear (for $M\left(\boldsymbol{k}, \boldsymbol{k}^{\prime}\right)$ and $\Delta \varepsilon\left(\boldsymbol{k}, \boldsymbol{k}^{\prime}\right)$, often piecewise constant) interpolants defined on a regular mesh in $k$-space. The underlying $k$-space mesh, $\left\{\boldsymbol{k}_{n}\right\}_{n}$, is usually cubic; interpolatory elements are then chosen to be either cubes [1], or tetrahedra (each cube being subdivided into five (equi-edge-length) or six (equivolume) tetrahedra [3]. Band structure, $\varepsilon\left(\boldsymbol{k}_{n}\right)$, gradients, $\nabla_{k} \varepsilon\left(\boldsymbol{k}_{n}\right)$, and scattering rates, $\boldsymbol{S}\left(\boldsymbol{k}_{n}\right)$, 
are then pre-computed and stored for interpolation during Monte Carlo execution. Since the $\varepsilon(\boldsymbol{k})$ relation possesses the (translation and point group) symmetry of the crystal, rather than store data at mesh points throughout the entire first Brillouin zone, it is often expedient to use a much smaller region of $k$ space known as an "irreducible wedge". For $\mathrm{Si}$, the irreducible wedge is $1 / 48$ of the Brillouin zone volume and $\varepsilon(\boldsymbol{k})$ for any $\boldsymbol{k}$ may be computed as $\varepsilon\left(\boldsymbol{k}_{I}\right)$, where $\boldsymbol{k}_{I}$ is a symmetryequivalent wavevector in the irreducible wedge.

Density of states and scattering rate integrals are thus reduced to sums of element integrals over the irreducible wedge, each element integral being relatively simple to evaluate. However, for the standard irreducible wedge in common use (described below), element integrals on some boundary planes and edges are cumbersome to evaluate. This occurs because the standard irreducible wedge cannot be precisely filled using a regular ${ }^{1}$ cubic or tetrahedral partition; along some boundaries there inevitably will be "overhang" of elements outside the irreducible wedge. In diamond-type semiconductors, elements intersecting [111] faces of the Brillouin zone fall into this category so that special care must be exercised to ensure accurate treatment of these contributions to Brillouin zone integrals.

\section{NEW IRREDUCIBLE WEDGE}

We have constructed a new irreducible wedge for use in diamond-type materials, which, compared with the standard irreducible wedge in common use (Fig. 1) considerably simplifies the handling of boundary element contributions to Brillouin zone integrals. This is especially true when the $\varepsilon(\boldsymbol{k})$ argument of the delta function is treated to quadratic order. Our irreducible wedge is depicted in Figure 2. Notice that the standard irreducible wedge lies entirely in the first Brillouin but has a

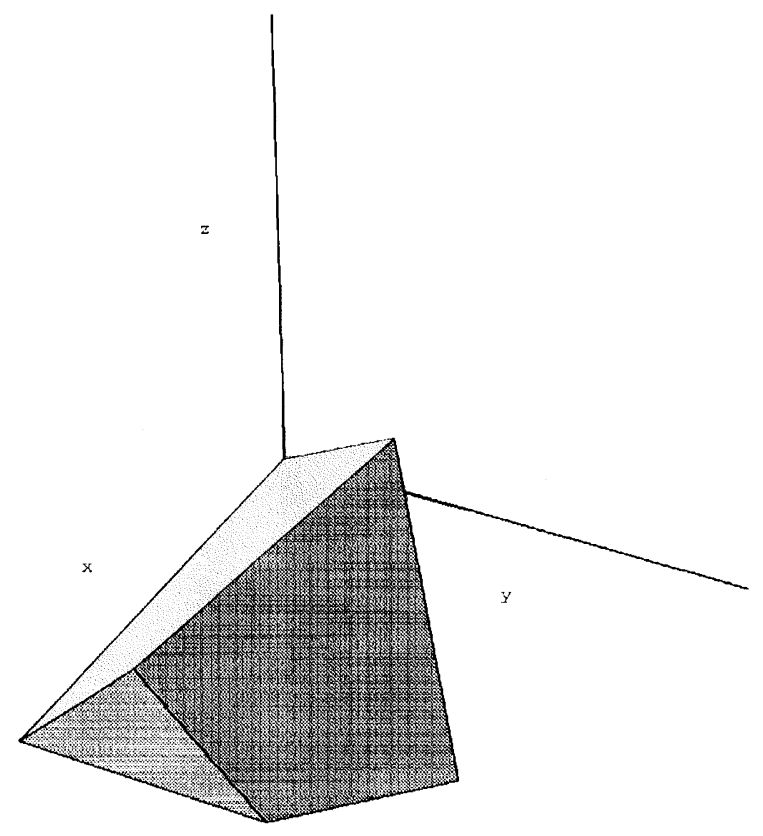

FIGURE 1 Standard irreducible wedge for Si.

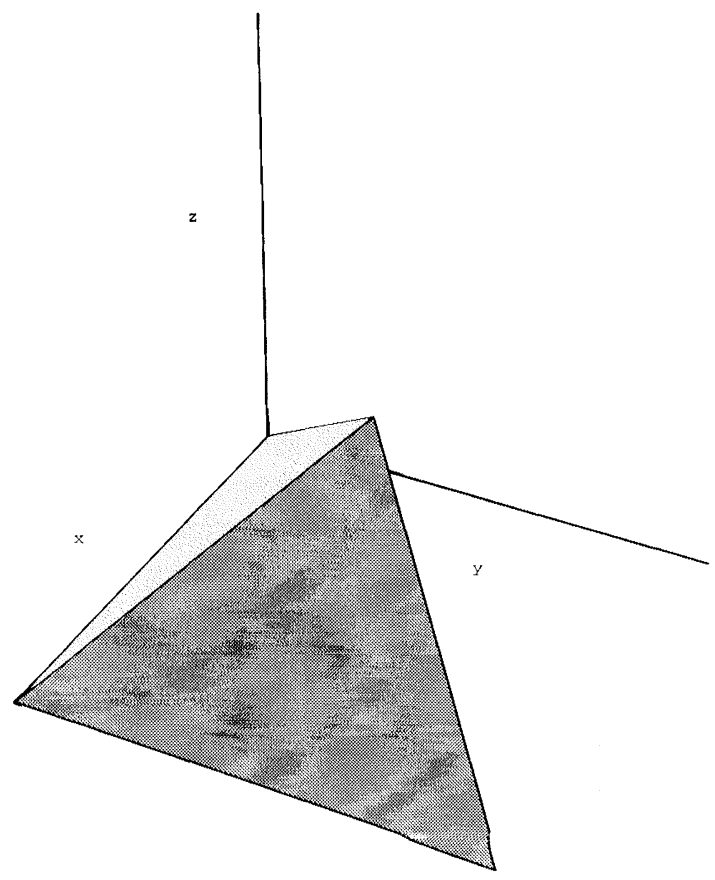

FIGURE 2 New irreducible wedge.

${ }^{1}$ By "regular" we mean identical up to translation and possibly a rotation. 
somewhat awkward geometry, while the irreducible wedge shown in Figure 2 (a tetrahedron of the same volume as the standard wedge) has simpler geometry but contains contributions from the first three Brillouin zones.

In contrast to the standard irreducible wedge, this new wedge allows a regular tetrahedral discretization in which there is essentially no distinction between boundary element and interior element contributions to integrals. That is, the new wedge may be precisely filled with a regular array of tetrahedral elements, while for the standard wedge, one inevitably finds elements intersecting the LUWK boundary plane to have portions extending outside the wedge. The presence of such "overhang", while posing no significant difficulties for element interpolations, can have a troublesome impact on the evaluation of integrals. The reason for this is as follows. Assuming a tetrahedral interpolation scheme, first note that evaluating interior element contributions to Brillouin zone integrals is relatively straight-forward (at least for the case where energy is interpolated only to linear order); tetrahedral element integrals reduce to simple surface integrals over planar triangles contained within the element. The difficulty for boundary elements not conforming to the wedge boundary arises from the need to include only that portion of the (constant energy or post-scattering state) surface which lies within the tetrahedron and also within the wedge. Clearly, no such difficulties occur for interior elements or for boundary elements conforming to the wedge boundary.

To illustrate the construction of this new irreducible wedge, refer to Figures 3-6. In Figure 3 we show an octahedron, which, if the vertices are sliced off by [100] planes, gives the first Brillouin zone (Fig. 4). The surface of the second Brillouin zone is shown in Figure 5. Note that both the first and second Brillouin zones are defined entirely by [100] and $\left[\frac{1}{2} \frac{1}{2} \frac{1}{2}\right]$ planes.

An examination of the second Brillouin zone reveals, by translation symmetry, that the portions of the octahedron vertices extending beyond the $\langle 100\rangle$ points may equivalently be subdivided into

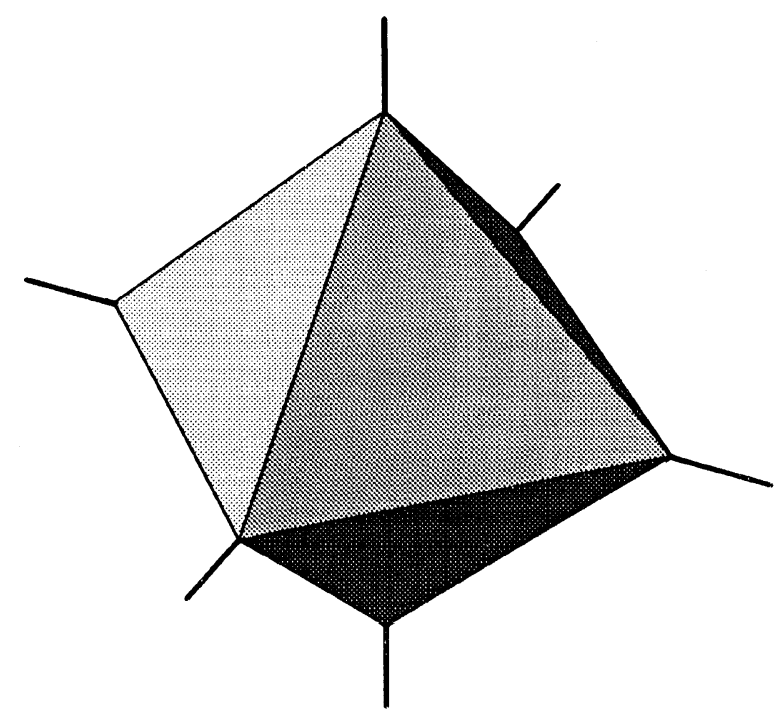

FIGURE 3 An octahedron.

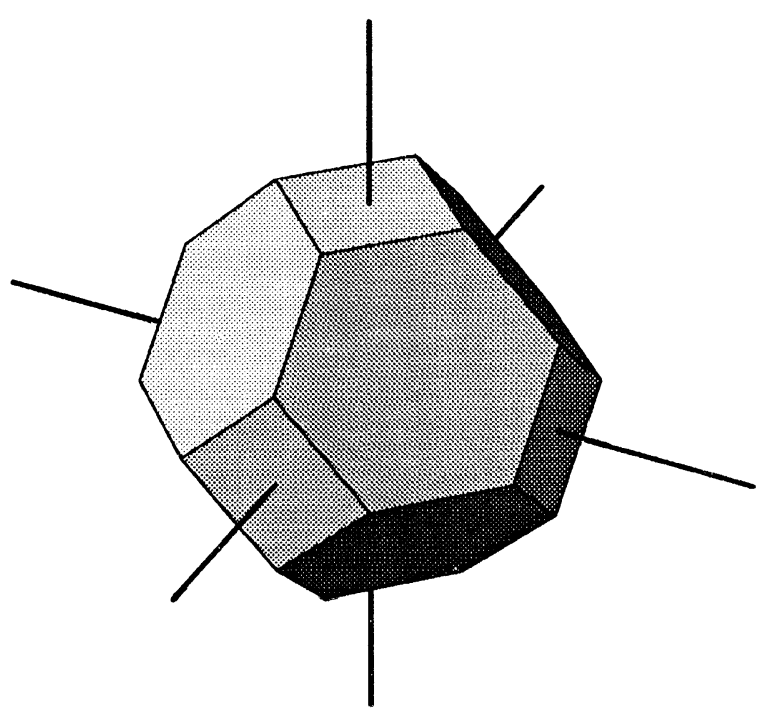

FIGURE 4 1st Brillouin zone of fcc lattice.

four identical tetrahedrons and moved pairwise from the $\langle 100\rangle$ axes to fill the "notches" present at the surface along $\langle 110\rangle$ directions. In carrying out this manipulation one converts the second Brillouin zone into a cube without changing the volume enclosed. The legitimacy of this transformation is readily established by appealing to the underlying space group symmetry. If we now 


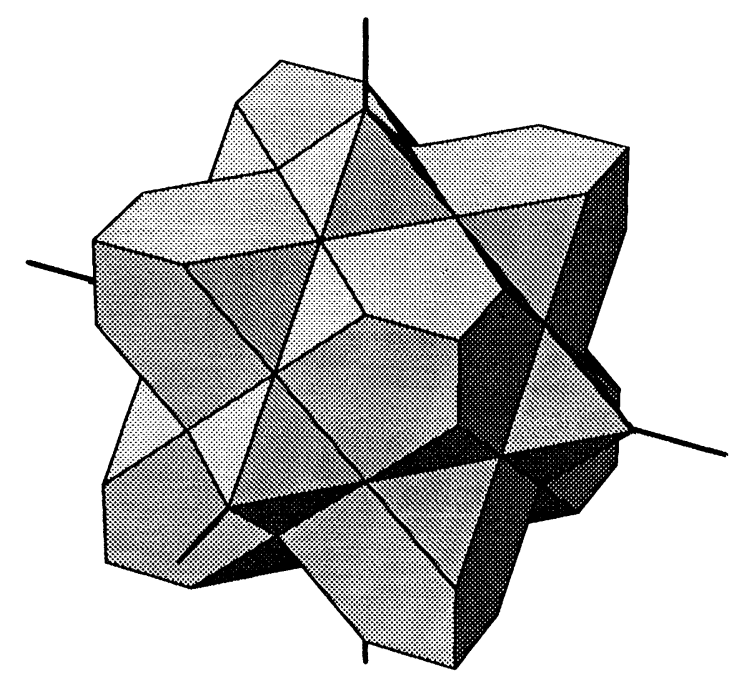

FIGURE 5 Surface of 2nd Brillouin zone of fcc lattice.

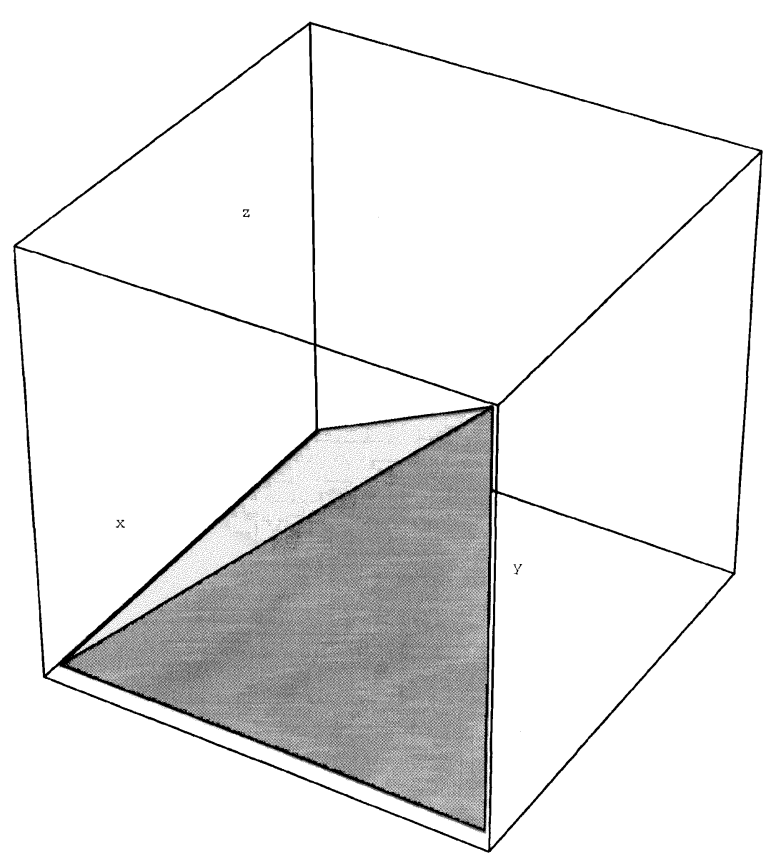

FIGURE 6 Wedge of modified 2nd Brillouin zone.

extend the standard irreducible wedge to pierce the faces of this cube, we obtain the right-tetrahedron shown in Figure 6. This irreducible wedge is exactly twice the volume of the standard irreducible wedge, containing $1 / 48$ of a zone in the first

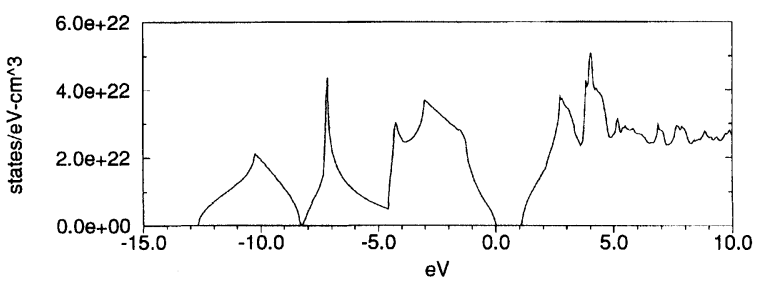

FIGURE 7 Density of states in $\mathrm{Si}$ computed using new irreducible wedge.

Brillouin zone and (the equivalent of ) $1 / 48^{\prime}$ th of a zone in the second and third Brillouin zones.

To reduce this tetrahedron to an equivalent irreducible wedge, we again appeal to symmetry. The tetrahedron shown in Figure 6 may be halved by cleaving along a $\left[\frac{1}{2} \frac{1}{2} \frac{1}{2}\right]$ plane; the two halves are identical by virtue of (diamond-type) reflection symmetry in [111] planes. We thus arrive at the new irreducible wedge shown in Figure 2.

\section{DENSITY OF STATES CALCULATION}

To illustrate the quality of integration provided by the new wedge, Figure 7 shows the results of a density of states calculation for the lowest ten (4 valence plus 6 conduction) bands in Si using a local, 113 plane-wave pseudopotential calculation [4] (without spin-orbit coupling included). The mesh used consists of 4000 tetrahedrons (946 distinct vertices) filling the new irreducible wedge. With $a$ denoting lattice constant, this corresponds to $0.05 \times$ $(2 \pi / a)$ increments for mesh points along $\langle 100\rangle$ directions. By using a regular tetrahedral discretization of the new wedge, one obtains remarkably smooth results with relatively little coding effort. For the present calculation the $\varepsilon(\boldsymbol{k})$ argument of the delta function is treated to linear order leading to "wiggles" near van Hove singularities [5].

\section{CONCLUSIONS}

We have defined a new $1 / 48$ Brillouin zone irreducible wedge applicable to diamond-type 
semiconductors. This new irreducible wedge facilitates accurate numerical evaluation of Brillouin zone integrals such as density of states and scattering rates by simplifying considerably the handling of boundary element contributions to such integrals.

\section{References}

[1] Fischetti, M. V. and Laux, S. E. (1988). “ Monte Carlo analysis of electron transport in small semiconductor devices including band-structure and space-charge effects", Phys. Rev. B, 38, 9721-9745.

[2] Callaway, J. (1991). Quantum Theory of the Solid State, Academic Press: Boston, Second Edn.

[3] Kunikiyo, T. et al. (1991). " A Monte Carlo simulation of anisotropic electron transport in silicon including full band structure and anisotropic impact-ionization model", J. Phys.: Condens. Mat., 3, 6721-6743.

[4] Chelikowsky, J. R. and Cohen, M. L. (1976). "Nonlocal Pseudopotential Calculations for the Electronic Structure of Seven Diamond and Zinc-blende Semiconductors", Phys. Rev. B, 14, 556-582.

[5] Wiesenekker, G. and Baerends, E. J. (1991). "Quadratic integration over the three-dimensional Brillouin zone", $J$. Phys.: Condens. Mat., 3, 6721-6743. 

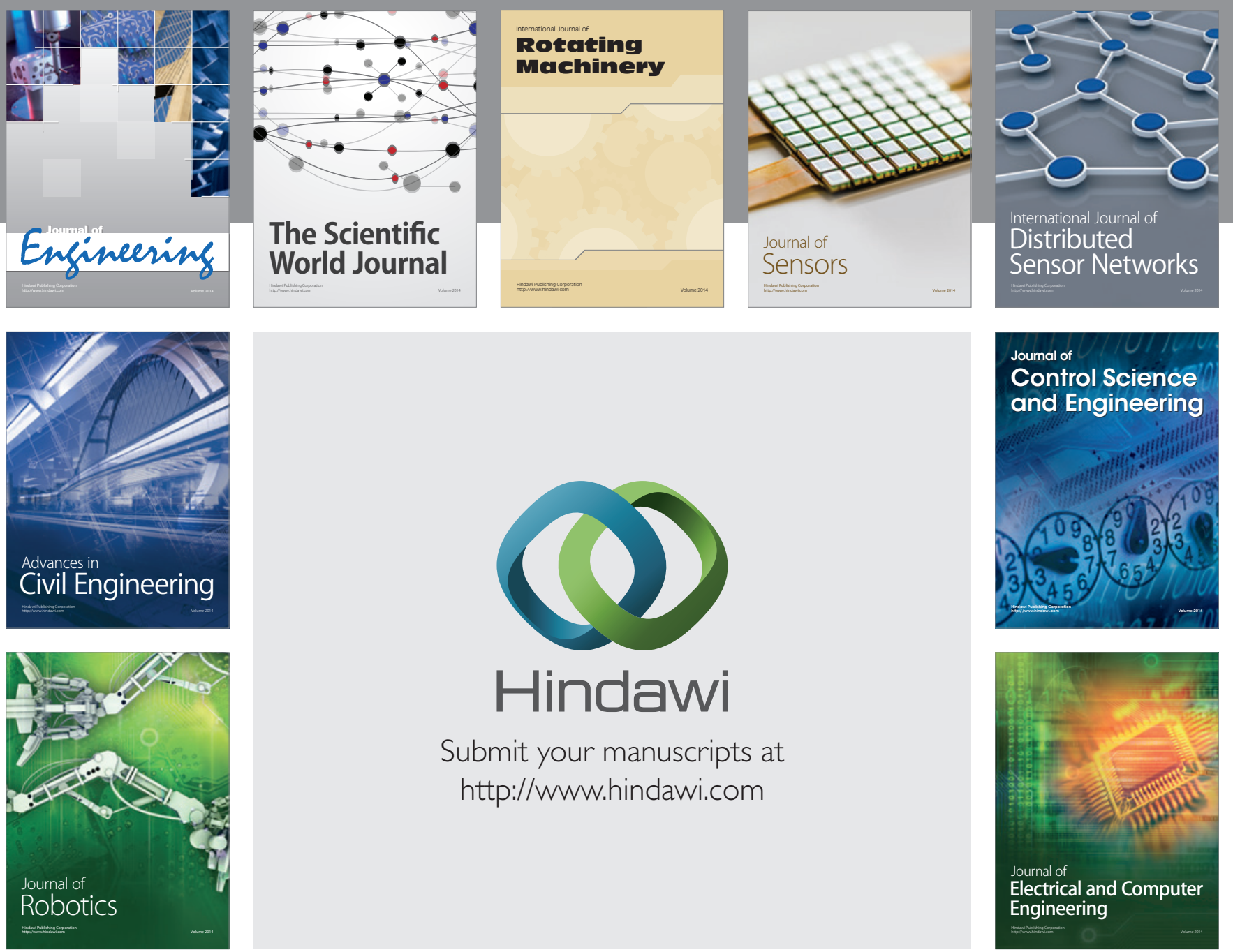

Submit your manuscripts at

http://www.hindawi.com
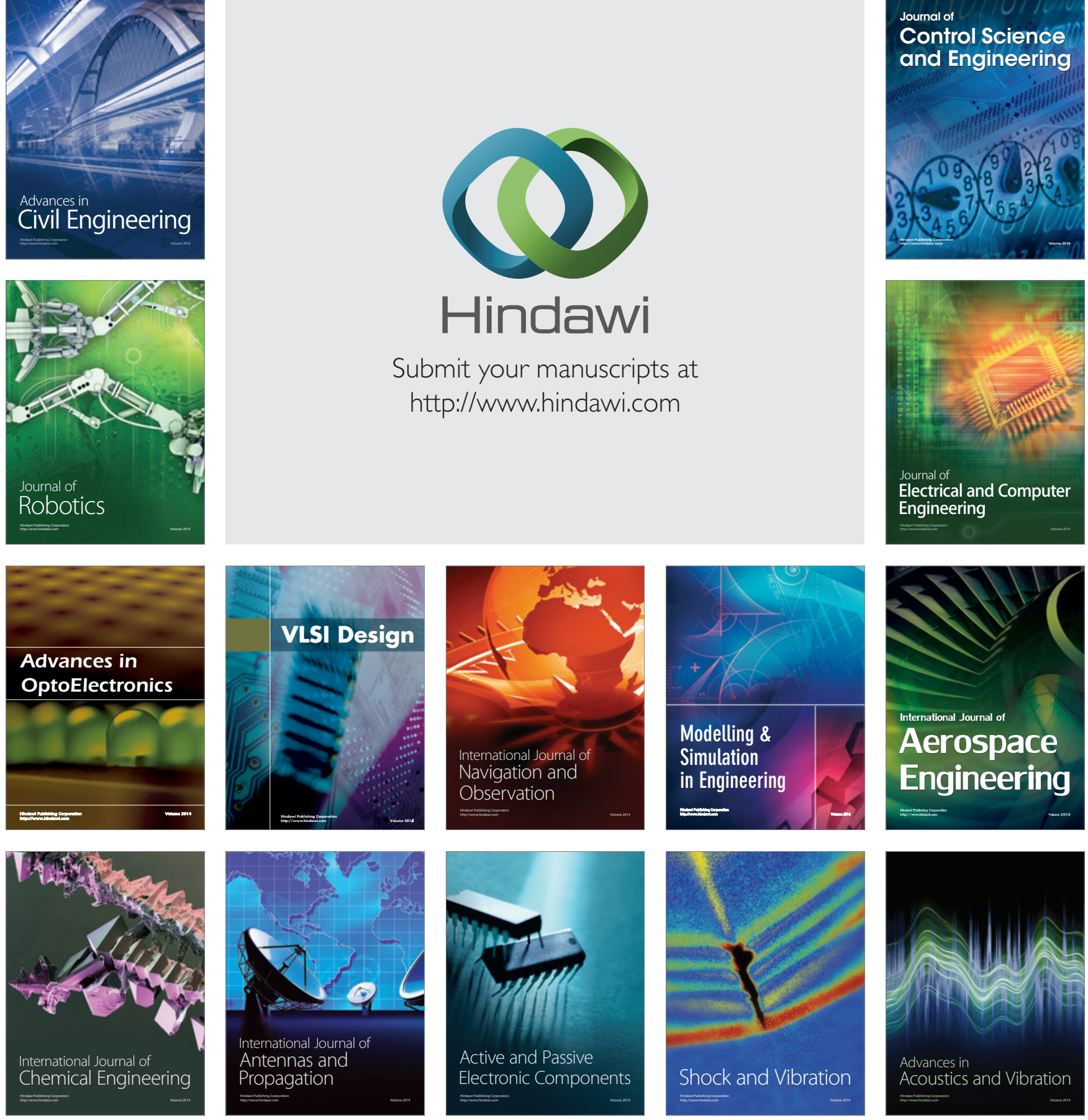